\title{
SENSORS FOR LOCATION-BASED AUGMENTED REALITY THE EXAMPLE OF GALILEO AND EGNOS
}

\author{
Alain Pagani $^{\mathrm{a}}$, José Henriques $^{\mathrm{a}}$, Didier. Stricker $^{\mathrm{a}}{ }^{\mathrm{b}}$ \\ ${ }^{a}$ German Research Center for Artificial Intelligence (DFKI) \\ ${ }^{\mathrm{b}}$ Technical University Kaiserslautern \\ alain.pagani@dfki.de
}

Commission I, WG I/5

KEY WORDS: Augmented Reality, Sensors, Galileo, Photogrammetry

\begin{abstract}
:
Augmented Reality has long been approached from the point of view of Computer Vision and Image Analysis only. However, much more sensors can be used, in particular for location-based Augmented Reality scenarios. This paper reviews the various sensors that can be used for location-based Augmented Reality. It then presents and discusses several examples of the usage of Galileo and EGNOS in conjonction with Augmented Reality.
\end{abstract}

\section{INTRODUCTION}

The concept of Augmented Reality (AR) has seen a fast-growing gain of interest in the last few years. Two decades ago, AR emerged as a specific field at the frontiers of virtual reality and computer vision. From this start, this topic continually expanded, benefiting from advances in various fields - computer vision, human-computer interaction or computer graphics to cite a few - and from constant technological innovation, making the necessary hardware both small and cheap. Today, AR seems to approach maturity, as simple AR-applications are commonly found in smartphones and are increasingly used for marketing operations. The term Augmented Reality is recognized as a meaningful concept for a large, non-professional public. Early theoretical research on AR attempted to provide a definition of Augmented Reality according to the then available state-of-the-art and future expectations. The concept of Virtuality Continuum was described as early as 1994 by Milgram and Kishino (Milgram and Kishino, 1994), and is still used today for describing modern AR applications. In 1997, Ronald Azuma defined AR as "a system that (1) combines real and virtual, (2) is interactive in real time and (3) is registered in 3D" (Azuma et al., 1997). Indeed, AR can be defined as a technology that supplements the real world with virtual (computer-generated) objects that appear to coexist in the same space as the real world. Figure 1 shows an example of Augmented Reality for small workspaces: here the virtual car is placed on top of a real book.

To achieve the necessary 3D registration, some sort of sensor is necessary. Because the aim of AR is often to augment an existing image, the sensor of choice is in many cases the camera that produced the image. Here the image itself is used as input data for computing the parameters to enable AR. However, other sensors can be used instead of - or in addition to - the camera for producing better results. In this paper, we review the various sensors that can be used for AR, starting from image analysis up to positioning using Global Navigation Satellite Systems (GNSS). We show that this last example is particularly well-suited for location-based AR scenarios.

The remainder of this paper is organized as follows: after a short description of the main tasks of Augmented Reality, we present a

\footnotetext{
${ }^{*}$ Corresponding author
}

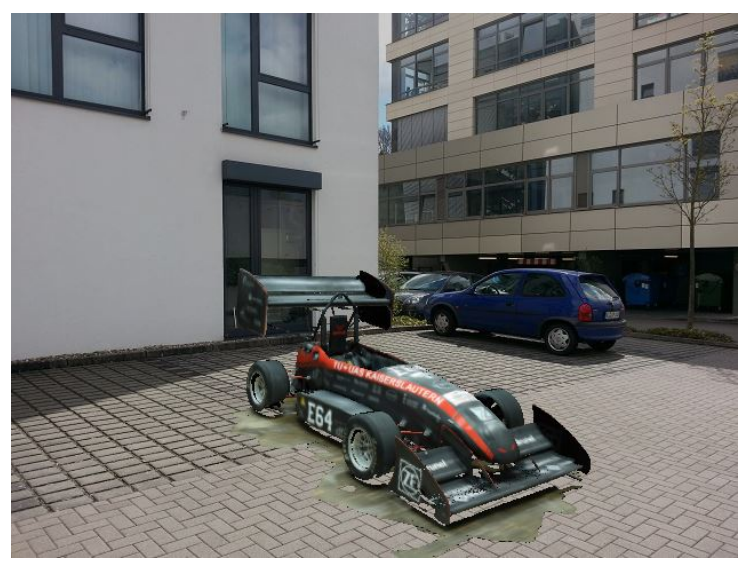

Figure 1: Example of AR application. Virtual car on a real parking lot

survey of sensors and related methods used for AR. We then take the example of Galileo and EGNOS to show of GNSS information can help in location-based Augmented Reality.

\section{AUGMENTED REALITY COMPONENTS}

In AR, the main task is to extend the user's senses (and in particular its vision) in order to present him/her additional information in form of virtual objects. Therefore, one important component in $\mathrm{AR}$ is a rendering engine capable of rendering virtual objects on top of an existing background image. For a realistic integration, care has to be taken to correclty model light sources, and to consider possible occlusions or shadows from the real objects onto the virtual ones. A study of the rendering component in AR is out of scope of this paper.

The second important component is a positional tracking module. In general, for the virtual object to appear as seamlessly integrated in the real environment, the 3D geometry of the supporting image has to be analyzed and well understood. In particular the exact position of the originating camera, its orientation and its internal parameters such as focal length, aspect ratio etc. 
must be known or computed from the image. In addition, some prior information about the underlying scene has to be known and modelled in order to correctly position the virtual objects. For example, the position of the ground plane has to be defined to serve as support for placing the virtual objects. Most if not all of the necessary information can be recovered by analysing the image content in a three-dimensional computer vision approach (Faugeras, 1993). The camera internal parameters - also called intrinsic parameters - can usually be computed once for all in a calibration procedure.

This procedure usually involves the acquisition of images of a calibration pattern. With sufficient images, it is possible to retrieve information such as focal distance, position of the principal point, ans skew factor. When assuming a non-zooming camera without automatic re-focus, these parameters can be considered constant so that the calibration procedure has to be done only once.

The problem of finding the camera position and oriention - also known as pose estimation problem - consists in finding the extrinsic parameters, i. e. the camera rotation and translation relative to a fixed coordinate system. In many applications, a real-time pose etimation technique is desirable. Here again, analysis of the image properties and of the underlying scene geometry can lead to very precise and fast results. This is particularly true when the geometry can by reconstructed beforehand using multiple images (Hartley and Zisserman, 2000), but it has also been shown that even a single image contains in some cases sufficient information for computing the pose through known objects, points of lines (Criminisi et al., 2000). When considering a complete video sequence, this pose estimation has to be repeated for each novel image. In that case, it can be useful to use the last known pose as initial guess for computing the pose of the camera for a novel image. This is the reason why pose estimation can be seen as tracking problem that can be solved using Bayesian filtering approaches (Thrun et al., 2005).

\section{SENSORS FOR POSE ESTIMATION}

The pose estimation problem - sometimes called tracking problem - amounts to track the viewer's movement with six degrees of freedom(6DOF): three variables $(\mathrm{x}, \mathrm{y}$, and $\mathrm{z}$ ) for position and three angles (yaw, pitch, and roll) for orientation. In many cases, a model of the real objects in the scene must be known in advance, but it does not need to be an accurate 3D model of the scene.

\subsection{Inside-out and outside-in pose estimation}

Generally the pose estimation methods can be split in two classe: the inside-out tracking methods and the outside-in tracking methods. In outside-in pose estimation, the user position is observed by an external sensor. In an optical context, this is done usually by adding some sort of markers to the head-worn device, and using a camera as external sensor. It can also use diverse other positioning methods. One major problem of outside-in tracking is that the range of movements of the user is limited to the field of view of the external camera. If the user moves outside of this field, or if the head rotation is too large, the tracking will break. In inside-out pose estimation, the sensor is placed on (or near to) the user and its pose is computed from the observations. Usually, one or more cameras are used, which are positioned so as to have an observation angle very close to the users eyes. Insideout tracking has several advantages compared to outside-in: first, the range of movements is not limited by the field of view of an
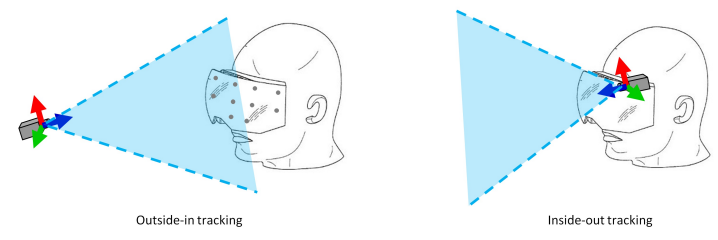

Figure 2: Outside-in vs Inside-out tracking approaches

external sensor, but merely by the ability to recognize the surrounding. Second, when using a camera for pose estimation in an inside-out configuration, the pose is computed from image measurements and can be optimized for small reprojection errors in the image plane. This means that the computed pose is optimal for a seamless integration of virtual objects in the real surrounding (i.e. the typical AR scenario). Third, when using an IMU, inside-out tracking allows for fusion of visual and inertial information in a very efficient and mathematically sound manner (Bleser and Stricker, 2008).

\subsection{Camera-based pose estimation}

Because the aim of AR is to augment an image, the camera has been traditionally used as primary sensor for pose estimation in AR. The camera is usually used in an inside-out tracking approach. By using one or several cameras, model-based approaches can recognise specific objects given an known model, or compute the relative movement between two images up to a scale factor. In order to create useful augmentations, for example for annotating a building with emergency exits, main stairways etc., a model of the scene or object to track has to be known in advance. This can also be useful to handle occlusions. When the scene can be prepared using non-natural objects, specific markers can be used in the so-called marker-based pose estimation method. If the preparation is not possible and only objects naturally present in the scene can be used, the technique is called markerless pose estimation.

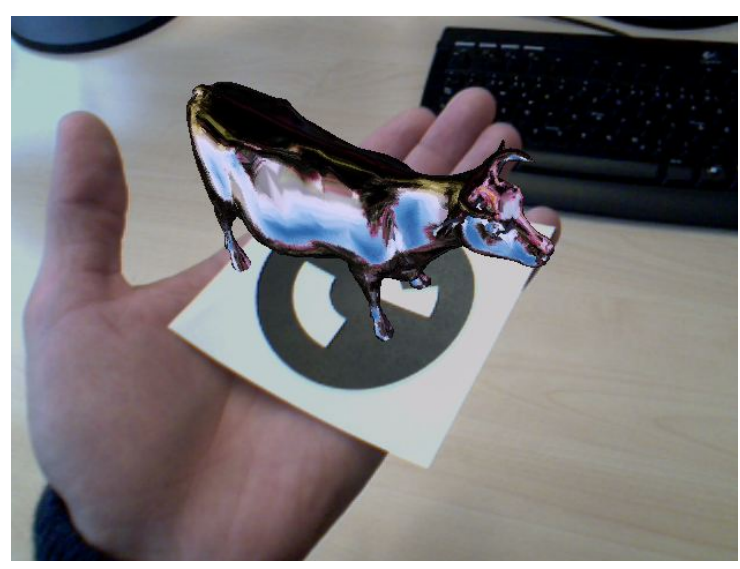

Figure 3: Example of a circular marker with augmentation

Marker-based pose estimation Historically, the first way of modelling the environment for pose estimation in AR was to use planar markers (Wagner and Schmalstieg, 2007). Most of the markers that can be found in the state of the art are square, black markers containing a code that can be used for identifying the marker. One major drawback of using square markers is that the computation of the camera pose relies on the precise determination of the four corners of the marker, which can be difficult in the case of occlusion. It has been shown recently that using circular markers, 
can be easier in the detection phase while providing a pose estimate that is more robust to noise (Koehler et al., 2011). Figure 3 shows an example of circular markers used in AR (Pagani et al., 2011).

Markerless pose estimation In some situations, standard markers cannot be used directly. This happens for example when the environment should look natural to the user in applications like augmented advertisement in magazines or outdoor scenarios. In this case, the objects naturally present in the scene can be modelled in 3D and be used as known objects for pose estimation. The general condition is that the objects have sufficient texture to allow for a precise detection of keypoints on their surface. Once $2 \mathrm{D}$ points with known $3 \mathrm{D}$ coordinates have been detected in the scene, the pose of the camera is computed by solving the Perspective from $n$ Points (PnP) problem (Hartley and Zisserman, 2000). Because these methods rely on point matching techniques, the input data is rarely exempt of outliers. For this reason, robust techniques such as RANSAC (Fishler and Bolles, 1981) can be used in the pose estimation process.

\subsection{Other sensors for pose estimation}

Even if a digital camera is often used for Augmented Reality, the use of an imaging device is not absolutely necessary. In an optical see-through setup, for example, no digital image is augmented, but rather the user's vision directly. This is made possible by using a see-through display, where only the virtual objects are rendered on a semi-transparent display, the background image being the real environment directly observed by the user. While seethrough displays offer a better immersion and increase the notion of presence (Schuemie et al., 2001), they come with a number of new challenges such as the necessity to calibration the eye of the user (position and intrinsic parameters) (Tuceryan and Navab, 2000), and an increase importance of tracking position and low latency between the head movements and the tracker's response.

Thus, besides the camera, several other sensors can be used for pose estimation, either alone or in addition to a camera in a hybrid approach.

3.3.1 Early trackers The first experiments of AR by Sutherland (Tamura, 2002) were done with an HMD that was tracked mechanically through ceiling-mounted hardware. Later on, Raab et al. introduced the Polhemus magnetic tracker, which was able to measure distances within electromagnetic fields (Raab et al., 1979). While this type of tracker had much impact on AR research, its use is made difficult in environment containing metallic parts or magnetic fields.

3.3.2 Inertial Measurements Units IMUs are small devices that contain accelerometers and gyroscopes. They usually deliver acceleration in translation and rotation at high frequency (100 fps), and can be used in hybrid systems in a fusion approach (Bleser and Stricker, 2008). The main problem of IMUs is the errors due to drift. In order to avoid these errors, the estimates must periodically be updated with accurate measurements. Nowadays, IMUs are commonly found in smartphones, although often not synchronized with the camera (Kim and Dey, 2009).

3.3.3 Radio-based trackers Radio frequency can be used for example with RFID chips that can be positioned in the environment to allow positioning (Willers, 2006). Complementary to RFID one can The wide-area IEEE $802.11 \mathrm{~b} / \mathrm{g}$ standards mainly used for wireless networking can also be used for tracking. The quality of the tracking highly depends on the number of chips of access points reachable in the environment (Bahl and Padmanabhan, 2000) (Castro et al., 2001).
3.3.4 Global Navigation Satellite Systems Global Navigation Satellite Systems (GNSS) are currently available or being implemented in many regions: The American Global Positioning System (GPS) (Getting, 1993), the Russian counterpart constellation Glonass, and the 30-satellite GPS Galileo, currently being launched by the European Union. GNSS has an accuracy of about 10-15 meters, which would not be sufficient for precise position tracking, but several techniques to increase the precision are already available. These techniques are referred to as GNSS augmentation. GNSS augmentation is a method of improving the navigation system's attributes, such as accuracy, reliability and availability, through the integration of external information into the calculation process. One can distinguish between satellite-based augmentation systems (SBAS), that uses additional satellite-broadcast messages, and ground-based augmentation systems (GBAS), where the prevision is increased by the use of terrestrial radio messages. An example of SBAS is the European Geostationary Navigation Overly Service (EGNOS), that supplements the GPS, GLONASS and Galileo systems by reporting on the reliability and accuracy of the positioning data. This reduces the horizontal position accuracy to the metre level. A similar system used in North America is the Wide Area Augmentation System (WAAS) that provides an accuracy of about 3-4 meters. For more accuracy, the environments have to be prepared with a local base station that sends a differential errorcorrection signal to the roaming unit: differential GPS yields 13 meter accuracy, while the real-time-kinematic or RTK GPS, based on carrier-phase ambiguity resolution, can estimate positions accurately to within centimeters (Van Krevelen and Poelman, 2010).

3.3.5 Hybrid trackers When several sensors can be used in the same setup, a hybrid method can be chosen. There are different ways to use multiple sensors. The most simple one is to use the available sensors sequentially, where the interpretation of one sensor's data depends on the measured data from the previous ones by using some heuristic. Another one is to fuse all available data using a Bayesian filter (Thrun et al., 2005), where the state is the pose to be estimated. For simple trackers, a Kalman filter can be used, but the complexity and in particular the non-linearity of the transition equations in the pose estimation problem often necessitates more elaborated models. For example, when dealing with multiple hypotheses, a particle filter can be useful.

\section{GALILEO AND EGNOS FOR OUTDOOR AUGMENTED REALITY}

In this section we present two possible uses of Galileo and EGNOS as sensors for location-based augmented reality through two scenarios: a city tourist guide and utility network augmentation.

\subsection{Example 1: City tourist guide}

In many AR applications, information should be delivered to the user at very specific locations. This type of location-based service makes the use of standard vision-based positional tracking for AR complicated, because the covered area is extremely large, and the environment cannot easily be prepared in advance. Moreover, even if large parts of the environment could be modeled (by generating an accurate 3D model of an entire city for example), it would not be practical to use the entire model as support of the pose estimation problem, because at a given point in time, only a tiny subpart of the model would be necessary for the pose estimation algorithm. In that case, the use of GNSS information can play a role of first coarse pose estimation in order to look for the right supporting model for a finer visual pose estimation. In 


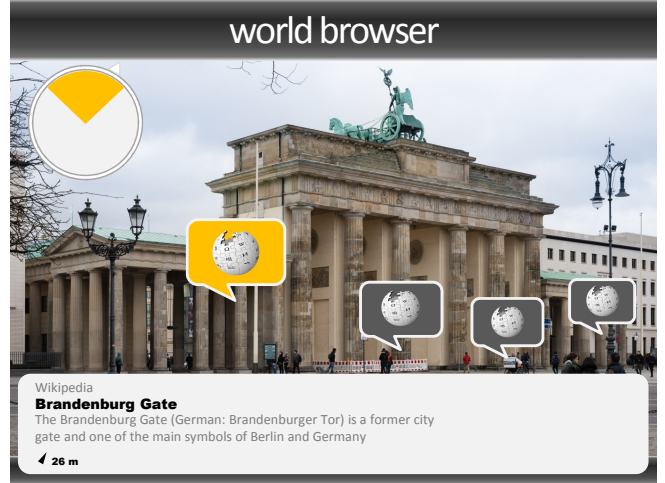

Figure 4: AR Labeling for world browsing. Exemplary application in the style of the Wikitude world browser

that case, the accuracy of standard GNSS such as Galileo (around $10 \mathrm{~m}$ ) could be sufficient for defining the coarse location).

A good application example is an AR-city guide using a smartphone. In that case, the user's coarse position (for example at the level of a street) can be obtained by Galileo positioning. If the smartphone contains a compass, the horizontal orientation of the device can also be obtained. Thus is it possible to know approximately where the user is and where he/she is looking at. With this simple approach, the system can already provide information such as the name of distant buildings or the direction and distance of other cities. This type of coarse pose estimation was commonly used in early smartphone AR applications such as Wikitude (Madden, 2011) and contributed to the success of AR for early adopters. Figure 4 shows an example of such a world browser application.

Another advantage of knowing the coarse position of the user is that further models that are required for a more precise pose estimation can be downloaded on demand, depending on the location of the user. For example, when the system detects that the user is in the front of a building, annotated pictures of this building can be downloaded and serve for a pixel-precise registration of live images with these pictures. It is this possible to deploy an AR application in an extremely large environment without shipping large models to all the users.

\subsection{Example 2: Utility network augmentation}

In other cases, the visual information might be difficult to model due to changing environments. For example, the European H2020 project LARA ${ }^{1}$ aims at developing a new mobile device for helping employees of utilities companies in their work on the field. The device to be developed called the LARA System consists of a tactile tablet and a set of sensors that can geolocalise the device using the European GALILEO system and EGNOS capabilities. The system itself is a mobile device for utility field workers. In practice, this device will guide the field workers in underground utilities to see what is happening underworld. The system is using Augmented Reality interfaces to render the complex 3D models of the underground utilities infrastructure such as water, gas, electricity, etc. in an approach that is easily understandable and useful during field work. The 3D information is acquired from existing 3D GIS geodatabases.

Due to the fact that the device is intended to be used in building sites, the models that are usually required for vision-based pose estimation are difficult to establish and maintain. In this case,

\footnotetext{
${ }^{1}$ http://lara-project.eu
}

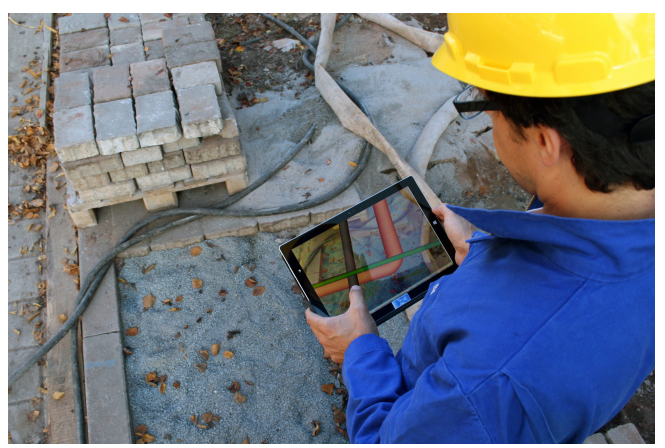

Figure 5: AR for utilities networks. Usage concept for the LARA system

it is therefore desirable to not rely on the camera as sensor for positional tracking.

Because the precision of about 10 meters is not sufficient, the use of a GNSS alone is not recommended for precise AR such as the one defined in the LARA scenario. Therefore the project makes use of the EGNOS system and other augmentations such as differential GPS (precision of less than 1 meter) and Precise Point Positioning (PPP) or Real Time Kinematics (RTK), with a precision of about $1 \mathrm{~cm}$. However, the obtained precision depends on the quality of the signal, the number of satellites currently observed and other factors. Therefore in this approach we estimate the obtained precision first using the EGNOS signal, and apply a heuristic to decide if we use directly the pose from the GNSS, or if we need to fuse this pose with visual information such as known points, or vanishing lines. All in all this system provides an accurate pose from GNSS when it is available and computes a better pose estimate with visual hints when the accuracy of the GNSS pose is not enough for AR. Figure 5 shows the usage concept for the LARA system.

\section{CONCLUSION}

In this paper, we reviewed different sensors that can be used for pose estimation in Augmented Reality. Even if the camera is the sensor of choice in most of the cases, we have seen that the use of Global Navigation Satellite Systems Information such as Galileo and EGNOS is convenient for location-based services, especially in outdoor scenarios. We provided two examples of use, the first one using coarse localization from GNSS to provide the metadata necessary for visual tracking, the second one relying on precision estimaton for defining the hybridation strategy to use.

\section{ACKNOWLEDGEMENTS}

The work leading to this publication has been partially funded by the European GNSS Agency through the Horizon 2020 project LARA, under grant agreement No GA 641460.

\section{REFERENCES}

Azuma, R. et al., 1997. A survey of augmented reality. Presence: Teleoperators \& Virtual Environments 6(4), pp. 355-385.

Bahl, P. and Padmanabhan, V. N., 2000. Radar: An in-building rf-based user location and tracking system. In: INFOCOM 2000. Nineteenth Annual Joint Conference of the IEEE Computer and Communications Societies. Proceedings. IEEE, Vol. 2, Ieee, pp. $775-784$. 
Bleser, G. and Stricker, D., 2008. Advanced tracking through efficient image processing and visual-inertial sensor fusion. Computer \& Graphics.

Castro, P., Chiu, P., Kremenek, T. and Muntz, R., 2001. A probabilistic room location service for wireless networked environments. In: Ubicomp 2001: Ubiquitous Computing, Springer, pp. 18-34.

Criminisi, A., Reid, I. and Zisserman, A., 2000. Single view metrology. International Journal of Computer Vision 40(2), pp. $123-148$.

Faugeras, O. D., 1993. Three-Dimensional Computer Vision: a Geometric Viewpoint. MIT Press.

Fishler, M. A. and Bolles, R. C., 1981. Random sample consensus: A paradigm for model fitting with applications to image analysis and automated cartography. Communications of the ACM 24(6), pp. 381-395.

Getting, I. A., 1993. Perspective/navigation-the global positioning system. Spectrum, IEEE 30(12), pp. 36-38.

Hartley, R. and Zisserman, A., 2000. Multiple View Geometry. Cambridge University Press.

Kim, S. and Dey, A. K., 2009. Simulated augmented reality windshield display as a cognitive mapping aid for elder driver navigation. In: Proceedings of the SIGCHI Conference on Human Factors in Computing Systems, ACM, pp. 133-142.

Koehler, J., Pagani, A. and Stricker, D., 2011. Detection and identification techniques for markers used in computer vision. In: Visualization of Large and Unstructured Data Sets - Applications in Geospatial Planning, Modeling and Engineering (IRTG 1131 Workshop), pp. 36-44.

Madden, L., 2011. Professional augmented reality browsers for smartphones: programming for junaio, layar and wikitude. John Wiley \& Sons.

Milgram, P. and Kishino, F., 1994. A taxonomy of mixed reality visual displays. IEICE Transactions on Information and Systems 77, pp. 1321-1329.

Pagani, A., Gava, C., Cui, Y., Krolla, B., Hengen, J.-M. and Stricker, D., 2011. Dense 3d point cloud generation from multiple high-resolution spherical images. In: Proceedings of the International Symposium on Virtual Reality, Archaeology and Cultural Heritage (VAST).

Project, n.d. Lara. http://lara-project.eu/.

Raab, F. H., Blood, E. B., Steiner, T. O. and Jones, H. R., 1979. Magnetic position and orientation tracking system. Aerospace and Electronic Systems, IEEE Transactions on (5), pp. 709-718.

Schuemie, M. J., Van Der Straaten, P., Krijn, M. and Van Der Mast, C. A., 2001. Research on presence in virtual reality: A survey. CyberPsychology \& Behavior 4(2), pp. 183-201.

Tamura, H., 2002. Steady steps and giant leap toward practical mixed reality systems and applications. In: VAR' 02.

Thrun, S., Burgard, W. and Fox, D., 2005. Probabilistic Robotics (Intelligent Robotics and Autonomous Agents). The MIT Press.

Tuceryan, M. and Navab, N., 2000. Single point active alignment method (spaam) for optical see-through hmd calibration for ar. In: Proceedings of the IEEE and ACM International Symposium on Augmented Reality (ISAR).

Van Krevelen, D. and Poelman, R., 2010. A survey of augmented reality technologies, applications and limitations. International Journal of Virtual Reality 9(2), pp. 1.
Wagner, D. and Schmalstieg, D., 2007. Artoolkitplus for pose tracking on mobile devices. In: Proceedingsofthe Computer Vision Winter Workshop (CVWW).

Willers, D., 2006. Augmented reality at airbus. In: International Symposium on Mixed \& Augmented Reality. 